\title{
Novel Fold and Putative Receptor Binding Site of Granulocyte-Macrophage Colony-Stimulating Factor
}

\author{
Kay Diederichs, Tom BoOne, P. Andrew Karplus*
}

Granulocyte-macrophage colony-stimulating factor (GM-CSF) stimulates the development of and the cytotoxic activity of white blood cells. Recombinant human GM-CSF has proven useful in the treatment of blood disorders. The structure of GM-CSF, which was determined at 2.4 angstrom resolution by $x$-ray crystallography, has a novel fold combining a two-stranded antiparallel $\beta$ sheet with an open bundle of four $\alpha$ helices. Residues implicated in receptor recognition, which are distant in the primary sequence, are on adjacent $\alpha$ helices in the folded protein. A working model for the receptor binding site is presented.

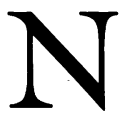

ATURAL GM-CSF IS A 127-AMINO acid residue trace glycoprotein (1, 2) that triggers the development of both granulocyte and macrophage colonies from hematopoietic progenitor cells (3). The availability of recombinant GM-CSF $(2,4,5)$ has allowed a much fuller characterization of its activities, and it is now clear that GM-CSF stimulates a wide variety of hematopoietic and nonhematopoietic cell types (6). As a therapeutic agent, GM-CSF has shown promise in the treatment of aplastic anemia, myelodysplastic syndromes, acquired immune deficiency syndrome, neutropenias, and chemotherapy-induced myelosuppression $(7,8)$. As a step toward understanding its structure-function rela-

K. Diederichs and P. A. Karplus, Section of Biochemistry, Molecular and Cell Biology, Cornell University, Ithaca, NY 14853

T. Boone, AMGEN, Thousand Oaks, CA 91320-1789.

*To whom correspondence should be addressed. tions, we have crystallized nonglycosylated recombinant human GM-CSF (9). Here we report the structure at $2.4 \AA$ resolution and define a putative receptor binding site.

The tertiary structure of GM-CSF is dominated by a bundle of four $\alpha$ helices and is distinctly nonspherical with dimensions of $20 \AA$ by $30 \AA$ by $40 \AA$ (Fig. 1). The four helices are all antiparallel and form a twisted open barrel, with the open edge filled in by strand $l$ of the antiparallel sheet. Each of the helices interacts with both of its neighbors around the barrel perimeter (Table 1 ) so that despite the presence of the $\beta$ sheet, GM-CSF can be compared with known four-helix bundle proteins (10). The topology of the bundle places helices A and B, and helices $\mathrm{C}$ and $\mathrm{D}$ across the barrel from each other rather than next to each other. This placement gives GM-CSF a double overhand topology (10), which has previously been seen for porcine growth hormone (11). Although interesting, the simi- 
larity to growth hormone does not extend to other key features of the GM-CSF fold, including the manner in which the helices pack together and the association of the two long overhand connections to build an antiparallel $\beta$ sheet. Growth hormone has long helices ( 20 to 30 residues) that pack with all interhelical angles close to $180^{\circ}$, whereas the GM-CSF helices are only between 10 and 16 residues long and cross at angles up to
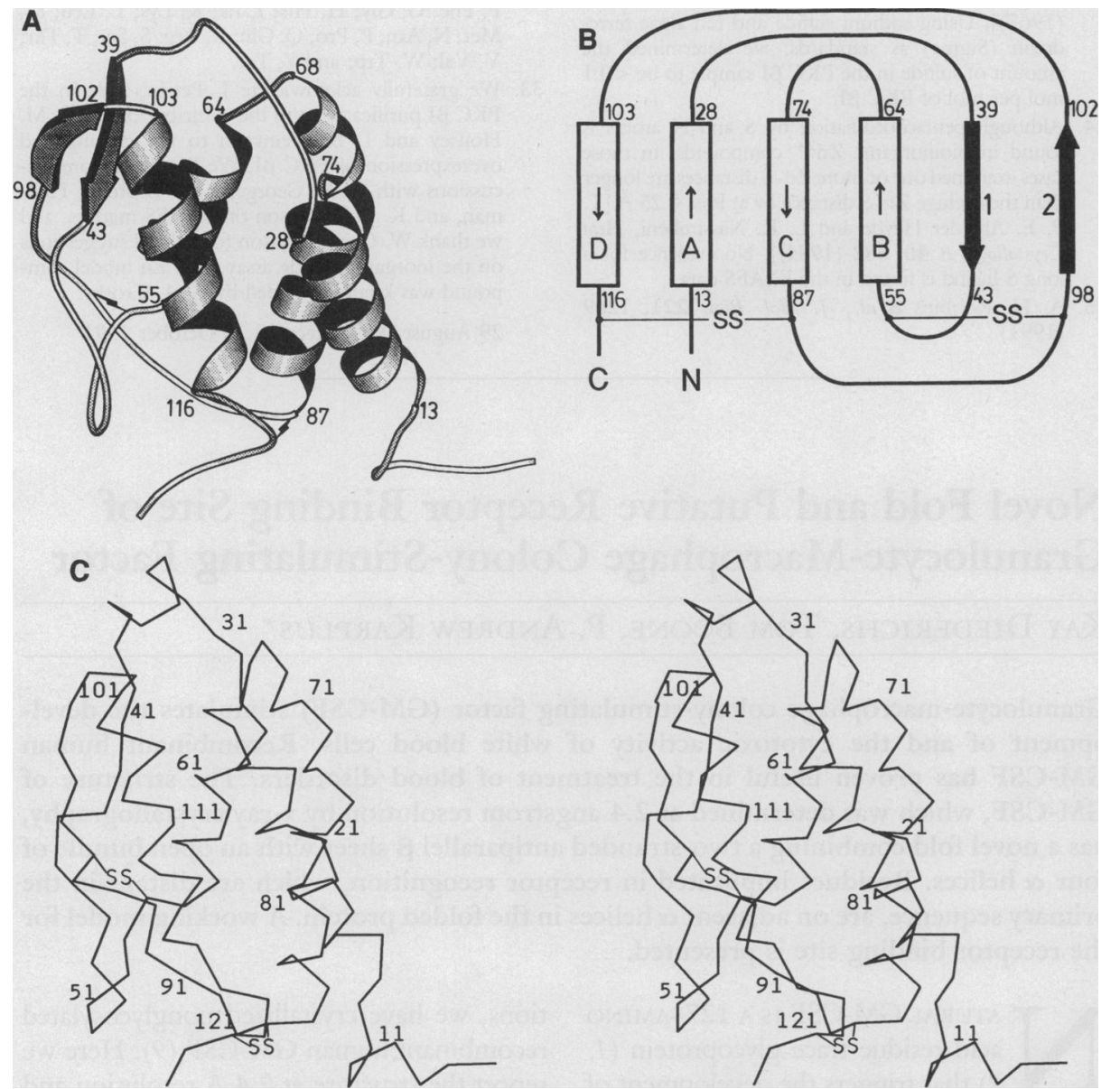

Fig. 1. The three-dimensional structure of GM-CSF. (A) A ribbon diagram (23) highlighting the secondary structural elements (24): helices are shown as spirals, $\beta$ strands as arrows, and disulfides as lightning bolts. (B) Topology diagram showing the $\alpha / \beta$-jellyroll topology. Rectangles represent helices $(\mathrm{A}, \mathrm{B}, \mathrm{C}$, and $\mathrm{D})$, and arrows represent $\boldsymbol{\beta}$ strands ( 1 and 2$)$. The $\mathrm{N}$ and $\mathrm{C}$ mark the termini, and the disulfides are indicated. (C) A stereo $\mathrm{C} \alpha$ diagram with every tenth residue labeled and disulfides indicated. The view is the same as in (A). The loops at residues 31 to 38 and 45 to 52 have high mobility. Crystals of recombinant human GM-CSF $\left(P 2_{1} 2_{1} 2_{1} ; a=47.6, b=59.1\right.$, and $\left.c=126.7 \AA\right)$ were grown and diffraction data were collected at room temperature on a San Diego Multiwire Systems detector (25) as described in the low-resolution structure determination (9). Two additional heavy-atom derivatives were found (mersalyl acid and $\mathrm{HgI}_{2}$ ) that allowed better experimental phases to be determined. The multiple isomorphous replacement phases were refined by iterative solvent flattening (26) combined with noncrystallographic symmetry averaging (9) to yield a $3.5 \AA$ resolution averaged electron density map that showed the characteristic spiral of the $\alpha$ helices and density for many side chains. One of the helices showed three large side chains pointing into the protein core and could be fit by residues 99 to 116, which include three Phe residues. No other parts of the sequence could be recognized in the electron density. After three rounds of model building and simulated annealing refinement (27) with the use of partial models, the electron density improved so that the true amino acid sequence could be assigned to the density. Five further cycles of model building and refinement were carried out and eventually used all of the data between 10 and $2.4 \AA$ resolution to yield an $R$ factor of 0.205. Individual atomic temperature factors were restrained, and the model has root-mean-square (rms) deviations from ideal geometry of 0.017 for bond lengths and $3.5^{\circ}$ for bond angles. The two molecules related by noncrystallographic symmetry have an rms deviation of $1.2 \AA$ for all atoms. The current model includes residues 5 through 123 of the protein and 64 bound water molecules. All model building was done on an Hewlett-Packard 9000/835 Workstation with the program FREIBAU (28). $\beta$-jellyrolls are right-handed. The two disulfide bonds in GM-CSF are both at the same end of the molecule and connect helix $B$ to strand 2 of the $\beta$ sheet and helix $C$ to the carboxyl terminus, respectively. Although there are no topological loop crossings, the loop from residues 43 to 54 penetrates the macrocycle formed by the carboxyl-terminal disulfide $\left(\mathrm{Cys}^{88}-\mathrm{Cys}^{121}\right)$ to result in a rare "threaded" topology (13). A conserved proline-rich sequence (residues 89 to 94; Fig. 2) adopts an extended conformation and makes key hydrogen bonds to stabilize the threaded interaction: the carbonyl of Pro ${ }^{92}$ hydrogen bonds to the amide of $\mathrm{Cys}^{54}$ and the carboxylate of $\mathrm{Glu}^{93}$ hydrogen bonds to amides of both $\mathrm{Glu}^{56}$ and $\mathrm{Thr}^{57}$ at the amino terminus of helix $B$.

Another notable feature of the fold is a stretch of $3_{10}$ helix from residues 68 to 73 . The Pro $^{76}$ residue interrupts the helical hydrogen-bonding pattern and causes a $30^{\circ}$ bend in the chain leading into helix $\mathrm{C}$. Since Pro $^{76}$ is not conserved across species (Fig. 2 ), it is unclear whether the bend is also present in murine GM-CSF or whether helix $\mathrm{C}$ might be longer.

The solvent accessibility of each residue in GM-CSF (Fig. 2) is useful for distinguishing those residues that contribute to structural integrity from those that may be directly involved in receptor recognition. We can use the GM-CSF structure to show that many mutation studies aimed at characterizing structure-function relations for GMCSF have been effective at locating structurally important regions rather than the receptor recognition site. A study of mutants in which various tripeptide deletions were made identified four regions in murine GM-CSF that were essential for activity (residues 18 to 22,37 to 44,54 to 64 , and

Table 1. Geometry of the GM-CSF helixpacking interactions. The interhelix packing angle $\Omega$ is the angle between the vectors defining the directions of the two helices (10). A negative angle means the front helix of the pair is rotated clockwise relative to the back helix. The separation is the closest approach of the two axial vectors. Presnell and Cohen (10) also assessed the compactness of a helical bundle by the amount of surface area that became buried as each helix associated with the other three helices. For GM-CSF these numbers are $24,21,37$, and $24 \%$ of the total accessible surface area of the four helices $\left(=4400 \AA^{2}\right)$, showing that the helices interact quite tightly with one another.

\begin{tabular}{lcc}
\hline Helix pair & $\begin{array}{c}\text { Separation } \\
(\AA)\end{array}$ & $\begin{array}{c}\Omega \\
(\text { degrees })\end{array}$ \\
\hline A:C & 8.4 & -147 \\
C:B & 9.5 & -171 \\
B:D & 11.4 & -137 \\
D:A & 10.3 & -148 \\
\hline
\end{tabular}


Fig. 2. Secondary structure and solvent accessibility in human GM-CSF (29). Line 1 documents the accessibility ("acc" in $\AA^{2}$ ) of each residue: $\bigcirc$ for acc $>50$; $O$ for $20<$ acc $<50$; $\bigcirc$ for acc $<$ 20. Line 2 gives the sequence of the human GMCSF used in this study (30), line 3 gives the sequence of murine (5) GM-CSF (dots

indicate identities with human GM-CSF) and line 4 shows the secondary structure: $H$, $\alpha$ helix; $E, \beta$ sheet; $G, 3_{10}$ helix; $T$, turn; and $S$, bend. The human numbering used throughout the text is given. Secondary structures and accessible surface areas were calculated with the program DSSPNOV (24). The gap in murine GM-CSF is placed so as to least disrupt the path of the chain

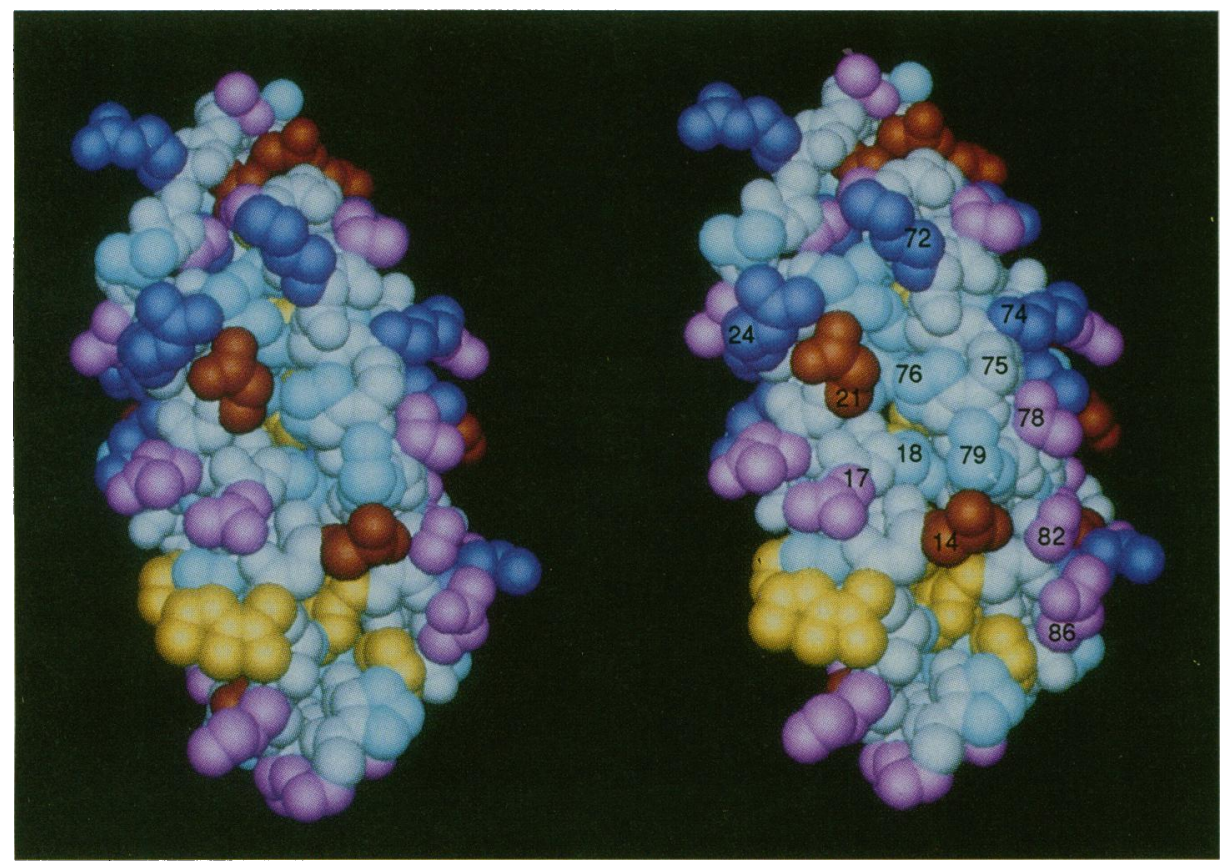

Fig. 3. A stereo view of the putative receptor binding site of GM-CSF. The view of GM-CSF shown is from the right-hand side of Fig. $1 \mathrm{C}$ and shows the narrow dimension of the molecule. The main chain is white, and side-chain colors are determined by residue type: apolar (G, A, P, C, V, I, L, and M), light blue; polar (S, T, N, and Q), purple; aromatic (H, F, Y, and W), yellow; negatively charged (D and $\mathrm{E})$, red; and positively charged ( $K$ and $R$ ), blue. Residues defining the putative recognition site are labeled by residue number. Asn ${ }^{27}$ is just visible to the left of $\mathrm{Arg}^{24}$, and a portion of $\mathrm{Arg}^{23}$ can be seen just below Asn ${ }^{27}$. Image generated by the program BRAGI (31).

96 to 118; human numbering) (14). These four regions correspond to helix $A, \beta$ strand 1 , helix $B$, and a stretch including $\beta$ strand 2 and helix D (Fig. 2). These regions are all likely to be critical for structural reasons and thus do not shed light on the receptor binding site. In another mutagenesis study, residues $17,21,38,41,46,59,63,65,104$, and 110 of murine GM-CSF (human numbering) were individually mutated to Pro residues (15). Again, comparison with the GM-CSF structure shows that only the mutations in $\alpha$ helices, where a Pro would cause structural disturbance (residues $21,59,63$, 66 , and 110), showed drastic activity loss. Other GM-CSF mutants that had near wildtype activity indicate that residues 1 to 16 , 67 to 70,121 to 127 , and the carboxyl- terminal disulfide do not play key roles in either stability or recognition $(14,16)$.

In contrast, an approach that takes advantage of the fact that human and murine GM-CSF are species-specific has identified key residues that are not structurally crucial. Assuming that equivalent regions of the murine and human GM-CSF are recognized by their receptors, Kaushansky et al. (17) used a series of chimeric human-murine GM-CSF molecules to show that residues 21 to 31 and 78 to 94 are important recognition regions. With residues 21 to 31 of murine GM-CSF replaced by the human counterpart, the hybrid no longer binds to the murine receptor, and the hybrid with residues 78 to 94 of human GM-CSF replaced by the murine sequence no longer binds to the human recep- tor. Because residues 88 to 94 are conserved between murine and human GM-CSF, the second implicated region can be redefined as 78 to 87. Two further constructs pointed to the importance of $\mathrm{Arg}^{23}$ or $\mathrm{Arg}^{24}$ or both, because an $\mathrm{Arg}^{23}$ to Gln mutant of human GM-CSF showed a $75 \%$ loss in specific activity and because inserting an Arg-Arg-Leu tripeptide after residue 22 of murine GMCSF (which puts it in registry with the human sequence) decreased its activity by $98 \%$. Further evidence consistent with the importance of residues 21 to 31 is the observation that deglycosylation of $\mathrm{Asn}^{27}$, which is adjacent to $\mathrm{Arg}^{24}$ on the surface of GM-CSF (Fig. 3), and $\mathrm{Asn}^{37}$ gives a 20 -fold increase in the specific activity of GM-CSF (18), as if one or both of the carbohydrate groups interfere with receptor binding.

Although some of the results seen for the hybrid proteins may be indirect effects due to changes in protein conformation, it is interesting that residues 21 to 31 and 78 to 87 are mostly in helix A and helix C, which are neighbors in the helical bundle (Fig. 1). Residue 76 is spatially adjacent to residue 21 , but as the helices are antiparallel, the two stretches of residues diverge from each other so that residue 87 is $35 \AA$ from residue 31 . Focusing on those implicated residues that are near each other allows us to present a putative receptor's view of the surface of GM-CSF centering on $\mathrm{Arg}^{24}$ and $\mathrm{Met}^{79}$ (Fig. 3). $\mathrm{Ala}^{18}, \mathrm{Gly}^{75}, \mathrm{Pro}^{76}$, and $\mathrm{Met}^{79}$ form a hydrophobic patch surrounded by the polar side chains of Glu ${ }^{14}, \mathrm{Asn}^{17}$, Glu ${ }^{21}, \mathrm{Arg}^{24}$, $\mathrm{Lys}^{72}, \mathrm{Lys}^{74}, \mathrm{Thr}^{78}, \mathrm{Ser}^{82}$, and $\mathrm{Gln}^{86}$. That residues 1 to 16 and the turn at 67 to 70 are not important for activity suggests the recognition site stops before $\mathrm{Glu}^{14}$ and does not extend above Lys $^{72}$. In addition to accounting for the available data, this proposed receptor recognition site also meshes nicely with ideas on protein-protein recognition, which suggest that hydrophobic groups are present to give strength to the interaction and hydrophilic groups to give the required specificity (19). Methodical mutagenesis of these surface residues should allow further delineation of the receptor recognition site or sites, and if receptor binding can truly be localized to residues from these two segments alone, it gives a reasonable hope that synthetic mimics of this surface may be developed.

The high-affinity (dissociation constant $K_{\mathrm{d}}=30$ pM) GM-CSF receptor on hematopoietic cells, which is formed when the $80-\mathrm{kD}$ receptor combines with a $120-\mathrm{kD}$ chain (20), has recently been shown to be a member of a family of structurally related receptors that includes the receptors for interleukin-2 and growth hormone (21). It is striking that GM-CSF, interleukin-2 (22), and growth hormone (11) have nonhomol- 
ogous structures and thus apparently have evolved their receptor binding activities independently. The one thing that appears common to these structures is the high helical content and the use of residues in $\alpha$ helices for receptor binding.

Note added in proof: The secondary structure and topology of the cytokine interleukin- 4 have been determined by nuclear magnetic resonance studies and it appears to have the same fold as GM-CSF (32).

\section{REFERENCES AND NOTES}

1. J. C. Gasson et al., Science 226, 1339 (1984).

2. G. G. Wong et al., ibid. 228,810 (1985)

3. S. C. Clark and R. Kamen, ibid. 236, 1229 (1987).

4. A. W. Burgess et al., Blood 69, 43 (1987); D. Metcalf, Science 254, 529 (1991)

5. N. M. Gough et al., Nature 309, 763 (1984).

6. J. C. Gasson, Blood 77, 1131 (1991).

7. M. A. S. Moore, Annu. Rev. Immunol. 9, 159 (1991).

8. K. M. Sakamoto, D. W. Golde, J. C. Gasson, J. Pediatr. 118, S17 (1991)

9. K. Diederichs, S. Jacques, T. Boone, P. A. Karplus, J. Mol. Biol. 221, 55 (1991).

10. S. R. Presnell and F. E. Cohen, Proc. Natl. Acad. Sci. U.S.A. 86, 6592 (1989).

11. S. S. Abdel-Meguid et al., ibid. 84, 6434 (1987).

12. J. S. Richardson, Adv. Prot. Chem. 34, 167 (1981).

13. T. Creighton, in Proteins: Structures and Molecular Properties (Freeman, New York, 1984), pp. 226227

14. A. B. Shanafelt and R. A. Kastelein, Proc. Natl. Acad. Sci. U.S.A. 86, 4872 (1989).

15. S. W. Altman, G. D. Johnson, M. B. Prystowky, J. Biol. Chem. 266, 5333 (1991).

16. I. Clark-Lewis et al., J. Immunol. 141, 881 (1988).

17. K. Kaushansky, S. G. Shoemaker, S. Alfaro, C. Brown, Proc. Natl. Acad. Sci. U.S.A. 86, 1213 (1989).

18. P. Moonen, J. J. Mermod, J. F. Ernst, M. Hirshi, J. F. DeLamarter, ibid. 84, 4428 (1987).

19. J. Janin and C. Chothia, J. Mol. Biol. 100, 197 (1976).

20. K. Hayashida et al., Proc. Natl. Acad. Sci. U.S.A. 87,9655 (1990).

21. J. F. Bazan, ibid. 87, 6934 (1990).

22. B. J. Brandhuber, T. Boone, W. C. Kenney, D. B. McKay, Science 238, 1707 (1987).

23. P. J. Kraulis, J. Appl. Crystallogr., 24, 946 (1991).

24. W. Kabsch and C. Sander, Biopolymers 22, 2577 (1983).

25. R. Hamlin, Methods Enzymol. 114, 416 (1985).

26. B. C. Wang, ibid. 115, 90 (1985).

27. A. T. Brünger, J. Kuriyan, M. Karplus, Science 235, 458 (1987)

28. P. A. Karplus, M. J. Daniels, J. R. Herriott, ibid. 251, 60 (1991)

29. Abbreviations for the amino acid residues are: $\mathrm{A}, \mathrm{Ala}$; C, Cys; D, Asp; E, Glu; F, Phe; G, Gly; H, His; I, Ile; K, Lys; L, Leu; M, Met; N, Asn; P, Pro; Q, Gln; R, Arg; S, Ser; T, Thr; V, Val; W, Trp; and Y, Tyr.

30. S. Miyatake, T. Otsuka, T. Yokota, F. Lee, K. Arai, EMBO J. 4, 2561 (1985).

31. D. Schomburg and J. Reichelt, J. Mol. Graphics 6, 161 (1988)

32. C. Redfield et al., Biochemistry 30, 11029 (1991).

33. The complete refined coordinate set for GM-CSF has been deposited in the Brookhaven Protein Data Bank (entry l GMF). K.D. thanks the Alexander von Humboldt Foundation (Bonn) for a postdoctoral fellowship. We thank S. Jacques for growing crystals and the Hewlett-Packard Corporation for donating the graphics workstation. Supported by a grant from the Cornell Biotechnology Program, which is sponsored by the New York State Science and Technology Foundation, a consortium of industries, the U.S. Army Research Office, and the $\mathrm{Na}$ tional Science Foundation. 